\title{
Small early gastric cancer with synchronous bone metastasis: A case report
}

\author{
ISAO FUJITA, TATSUYA TOYOKAWA, TAKAFUMI MAKINO, \\ KATSUNORI MATSUEDA, SHIZUMA OMOTE and JOICHIRO HORII \\ Department of Gastroenterology, National Hospital Organization, \\ Fukuyama Medical Center, Fukuyama, Hiroshima 720-8520, Japan
}

Received March 17, 2019; Accepted December 2, 2019

DOI: $10.3892 / \mathrm{mco} .2020 .1985$

\begin{abstract}
Bone metastasis during the early stages of gastric cancer is rare, and synchronous bone metastasis is even less common. The present report outlines a case of a small early gastric cancer, which was detected due to bone metastasis. A 63-year-old man was referred to Fukuyama Medical Center with back pain and anorexia of 2 weeks' evolution. MRI revealed multiple metastatic lesions in the thoracic and spinal bone. Fluorodeoxyglucose positron emission tomography revealed focal uptake in the lesser curvature of the stomach and in the spinal bone, pelvic and thigh bone, but uptake was not detected in the stomach. Esophagogastroduodenoscopy revealed a $10 \mathrm{~mm}$ slightly elevated lesion with a central depression in the middle-third of the stomach. Endoscopic ultrasonography confirmed that the tumor was confined to the mucosa. A biopsy specimen acquired from the gastric lesion indicated signet-ring cell carcinoma, and the specimen acquired from the lumbar spine revealed cell aggregation such as that found in signet-ring cell carcinoma. The patient received first-line chemotherapy with $\mathrm{S}-1$ and cisplatin, and second-line chemotherapy with nab-paclitaxel. However, the patient died 120 days after consultation at Fukuyama Medical Center.
\end{abstract}

\section{Introduction}

Early gastric cancer (EGC) is defined by the Japanese Gastric Cancer Association as cancer that is confined to the mucosa or submucosa, regardless of the presence of lymph node metastasis with a 5-year survival rate $\geq 85 \%$ (1-4). However, when EGC with bone metastasis develops into disseminated bone marrow carcinosis combined with disseminated intravascular

Correspondence to: Dr Isao Fujita, Department of Gastroenterology, National Hospital Organization, Fukuyama Medical Center, 4-14-17 Okinogami-cho, Fukuyama, Hiroshima 720-8520, Japan E-mail: i.fujita777dr@gmail.com

Key words: early gastric cancer, bone metastasis, fluorodeoxyglucose positron emission tomography coagulation, prognosis is generally poor. Bone metastasis is common in patients with advanced breast, lung, kidney and prostate cancer but not in patients with gastric cancer. In gastric cancer, the incidence of bone metastasis is higher in advanced cancer than in early lesions (5).

Fluorodeoxyglucose positron emission tomography (FDG-PET) is considered to be useful for staging malignant tumors, including lung, esophagus, and colon cancer, and for identifying metastatic lesions. However, in patients with gastric cancer, FDG-PET is not useful for the detection of regional lymph nodes or T1 tumors (6).

We present the case of a small EGC with bone metastasis, which was diagnosed by esophagogastroduodenoscopy (EGD) and FDG-PET. Written informed consent was obtained from the patient for the publication of the case details and associated images.

\section{Case report}

In 2014, a 63-year-old Japanese man was referred to our hospital with back pain and anorexia of 2 weeks' evolution. He had no relevant personal or family history. His height was $169 \mathrm{~cm}$, weight was $71 \mathrm{~kg}$. His laboratory results were as follows: lactate dehydrogenase (LDH), 779 IU/1; alkaline phosphatase, 347 IU/l; serum calcium level, $12.6 \mathrm{mg} / \mathrm{dl}$; and carcinoembryonic antigen and carbohydrate antigen 19-9 levels, 34.11 and $5.64 \mathrm{ng} / \mathrm{ml}$, respectively. Enhanced CT revealed slight lymph node enlargement in the lesser curvature of the stomach (Fig. 1). Magnetic resonance imaging demonstrated multiple metastatic lesions in the thoracic and spinal bone (Fig. 2). FDG-PET was performed and revealed focal uptake in the lesser curvature of the stomach and the spinal, pelvic, and thigh bone but did not detect uptake in the stomach (Fig. 3). EGD revealed a $10 \mathrm{~mm}$ slightly elevated lesion with a central depression in the middle-third of the stomach. Endoscopic ultrasonography (EUS) confirmed that the tumor was confined to the mucosa (Fig. 4). A biopsy specimen acquired from the lesion indicated signet-ring cell carcinoma, and histological biopsy from the lumbar spine revealed cell aggregation such as that noted in signet-ring cell carcinoma (Fig. 5). The final diagnosis was confirmed to be EGC with synchronous multiple bone metastasis. The patient was treated using elcatonin and zoledronic acid for hypercalcemia, and 
chemotherapy ( $\mathrm{S}-1+$ cisplatin) for the gastric cancer was initiated. S-1 (100 mg/body/day) was given orally twice daily for the first 3 weeks of a 5-week course. CDDP was given as an intravenous infusion of $100 \mathrm{mg} /$ body/day on day 8 of each course. After the initiation of therapy, his LDH and serum calcium levels decreased and pain was alleviated. However, two months after the initiation of therapy, his LDH level increased again and therefore he was administered second-line chemotherapy using nab-paclitaxel. After being administered two courses of nab-paclitaxel (440 mg/body on day 1, every 21 days), he complained of dyspnea. CT revealed interstitial pneumonia (broad ground-glass opacity in both lungs); thus, since we diagnosed drug-induced pneumonia caused by nab-paclitaxel, we discontinued nab-paclitaxel and initiated prednisolone. These chemotherapies were authorized by Japanese Gastric Cancer Association and Pharmaceuticals and Medical Devices Agency of Japan (7). Although interstitial pneumonia improved, the patient's general condition worsened during that time, and we were unable to administer a new anticancer drug. And the patient died 120 days after consultation at our hospital.

\section{Discussion}

Bone metastasis is common in prostate, breast, lung, kidney and thyroid cancers, but it is rare in gastric cancer. Yoshikawa et al reported that bone metastasis occurred in $0.7-1.4 \%$ of gastric cancer patients (8). Park et al reported that bone recurrence after curative resection of gastric cancer was detected in $1.8 \%$ of the cases, and the incidence of bone recurrence was significantly higher in advanced gastric cancer (3.5\%) than in early lesions (0.4\%) (5). Choi et al reported that the incidence of bone metastasis was $45.3 \%$ in gastric cancer cases, including patients with non-curative resection, and Mori et al stated that bone metastasis occurred in $15.9 \%$ of gastric cancer cases in autopsy studies $(9,10)$. Thus, there are a variety of reports on the incidence of bone metastasis with gastric cancer, and the rate of bone metastasis may be higher than we believed. Kobayashi et al suggested that a reason that asymptomatic bone metastasis could be underestimated is that examination by bone scintigraphy is not a routine clinical practice (11). In addition, Park et al, reported that the rate of bone metastasis varied depending on the progression of gastric cancer (5).

Gurzu et al (12) reviewed the literature on metastasis with early gastric cancer in PubMed from 1983 to 2015. Only 79 cases of EGC with bone metastasis had been published. The time interval between the diagnosis of EGC and bone metastasis was reported in 47 cases out of 79 cases, and 17 cases (36.2\%) involved synchronous bone metastasis (12). Our case of early gastric cancer with synchronous bone metastasis found taking the back pain as an opportunity, was considerably rare.

Park et al also reported that the risk factors for recurrence of bone metastasis after curative resection of gastric cancer included depth of invasion and lymph node metastasis (5). Similarly, Kobayashi et al reviewed Japanese case reports to investigate the characteristics of bone metastasis from EGC, and they suggested that risk factors for bone metastasis from EGC included depressed-type signet-ring cell carcinoma, poorly differentiated carcinoma, and/or the likely involvement of lymph node metastasis (11). In our case, although

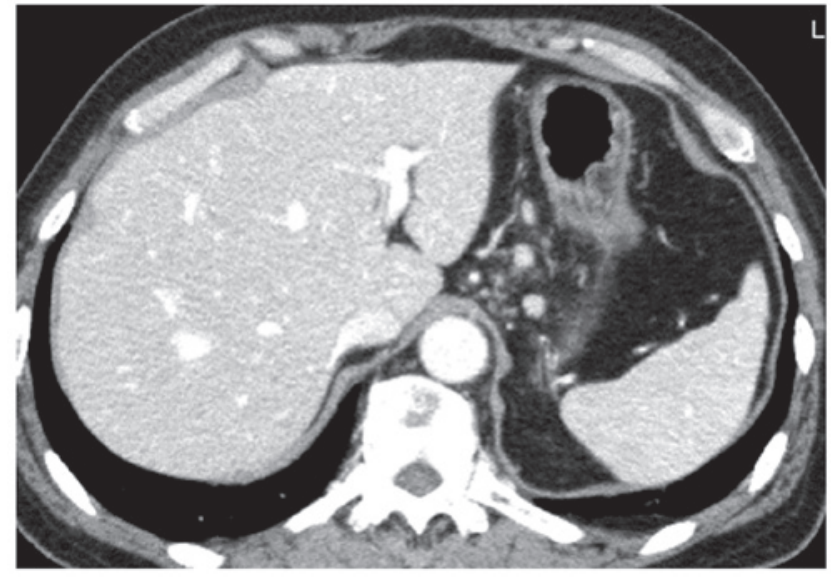

Figure 1. Enhanced CT scan revealed slight lymph node enlargement in the lesser curvature of the stomach.
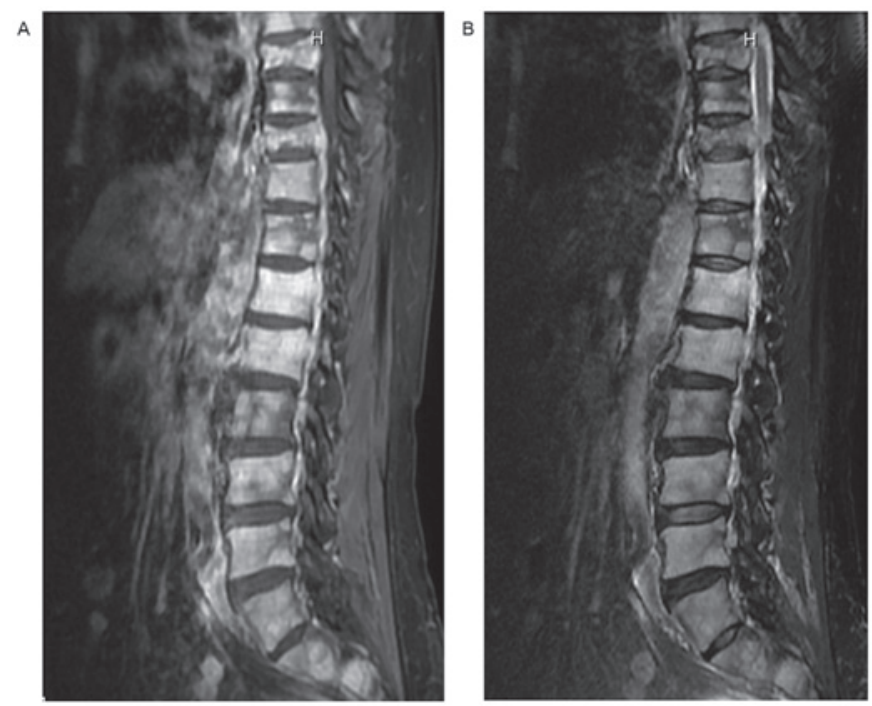

Figure 2. (A) T1 and (B) T2 MRI demonstrated multiple metastatic lesions in the thoracic and lumbar vertebrae.

histological examination with specimen removal was not performed, we diagnosed early gastric cancer by EGD and EUS. Our case was that of a slightly elevated lesion with a central depressed type signet-ring cell carcinoma with suspected lymph node metastasis, matching the characteristics described above.

Bone scintigraphy is frequently used for the diagnosis of bone metastasis. Recently, FDG-PET has been reported to be useful for the detection of primary malignant lesions as well as for distant metastasis including bone metastasis. However, the detection of gastric cancer by FDG-PET is believed to be determinant, given that the results are based on physiological accumulation in a stomach wall and the depth and histopathological type of the tumor. Dassen et al reported that the sensitivity of FDG-PET was 26-63\% for early gastric cancer and $93-98 \%$ for advanced gastric cancer (13). In addition, it is thought that FDG-PET has a lower sensitivity in detect diffuse type, mucinous adenocarcinoma or signet-ring cell carcinoma than other histological types, due to lower glucose transporter 1 (GLUT1) expression. The lesion is 
A

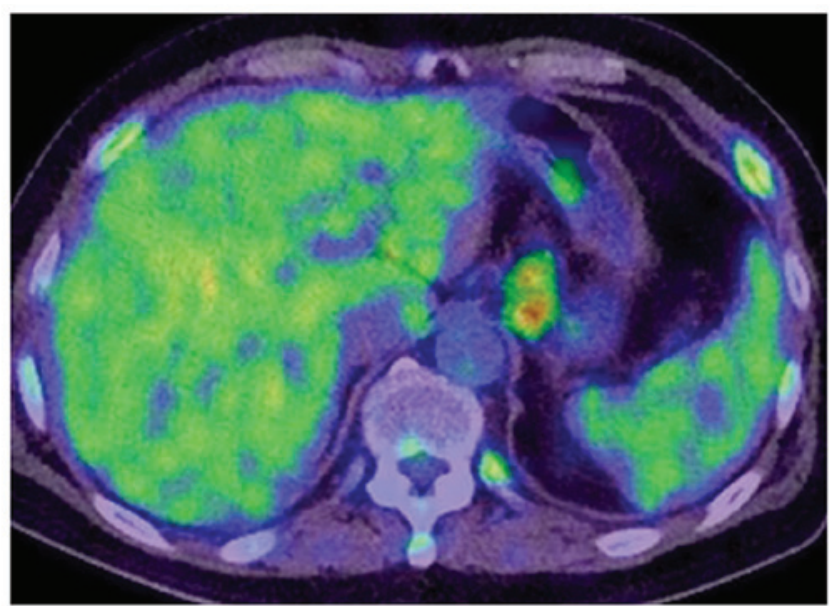

B
C

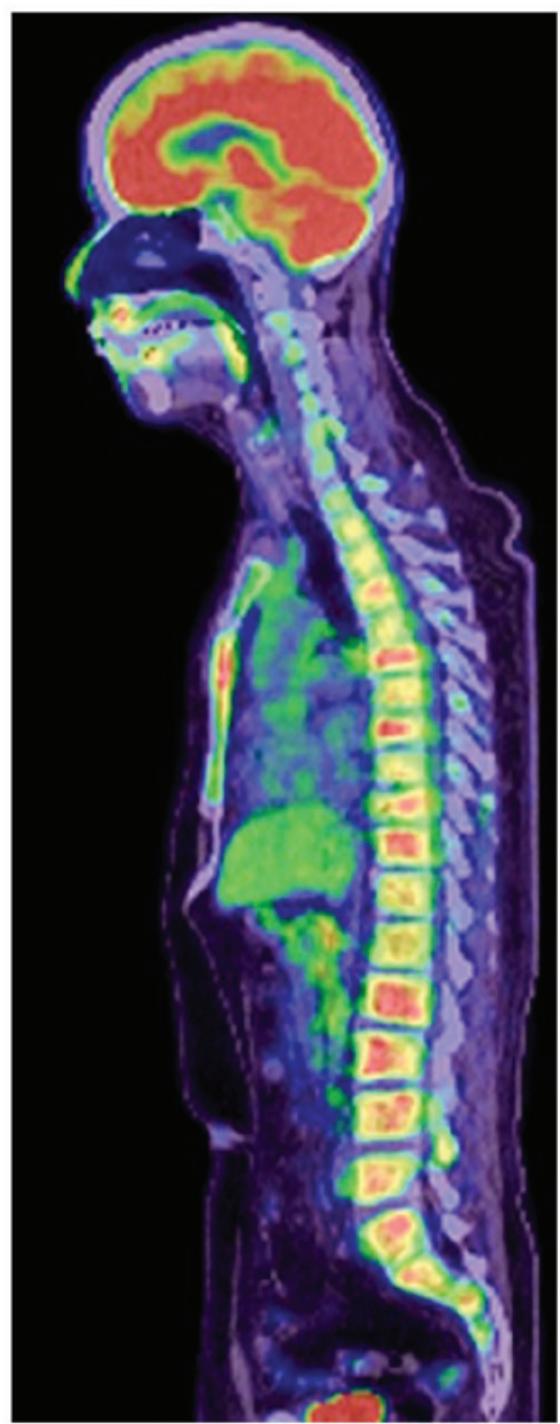

Figure 3. FDG-PET revealed focal uptake in the lesser curvature of the (A) stomach and (B and C) the spine, pelvic and thigh bone, but did not detect uptake in the stomach. FDG-PET, fluorodeoxyglucose positron emission tomography.

visually recognized as accumulation of a malignant neoplasm on FDG-PET. Cellular FDG uptake is related to GLUT1 expression, and GLUT1 is frequently overexpressed in malignant tissue. Kawamura et al analyzed GLUT1 expression in gastric neoplasms and reported that $30 \%$ of gastric cancers were positive for GLUT1 expression. In addition, signet-ring cell carcinoma and mucinous adenocarcinoma showed very low positive values for GLUT1 expression (2 and 6\%, respectively) (14). Yamada et al and Alakus et al evaluated whether cohesive carcinoma (papillary adenocarcinoma, tubular adenocarcinoma, and solid-type poorly differentiated adenocarcinoma) were more easily detectable than non-cohesive carcinoma, signet-ring cell carcinoma, and non-solid type poorly differentiated adenocarcinoma. They found that GLUT1 expression was the most important factor for determining the degree of FDG uptake $(15,16)$.

Previous studies have shown that the sensitivity and specificity of FDG-PET for the detection of bone metastasis in gastric cancer were $30-100 \%$ and $25-99 \%$, respectively (17-19). Shimada et al reviewed recent publications to evaluate the clinical utility of FDG-PET for gastric cancer and reported that FDG-PET had high specificity for detecting distant metastasis including bone metastasis, but it was not useful for the detection of regional lymph nodes or T1 tumors (6). We believe that FDG-PET is useful for bone metastasis cases because it is possible to detect a distant metastasis including a bone metastasis or a malignant tumor anywhere in the body except for early gastric carcinoma.

EGC with signet-ring cell carcinoma has a possibility of metastasizing to the bone, even if the tumor is small; as Kang et al reported, EGC that presented the small flat erosions with bone marrow metastasis, and it has features that are difficult to identify by FDG-PET (20). Therefore, when bone metastasis is detected but a primary lesion is not detected by FDG-PET, early gastric cancer should be anticipated, and a careful EDG should be performed.

Chemotherapy or radiation treatment for pain reduction are common treatments for gastric cancer with bone metastasis. Hironaka et al reported the effectiveness of sequential methotrexate and 5-fluorouracil for gastric cancer with bone metastasis (21). However, in the 2008 S-1 plus cisplatin versus S-1 alone for first-line treatment of advanced gastric cancer 

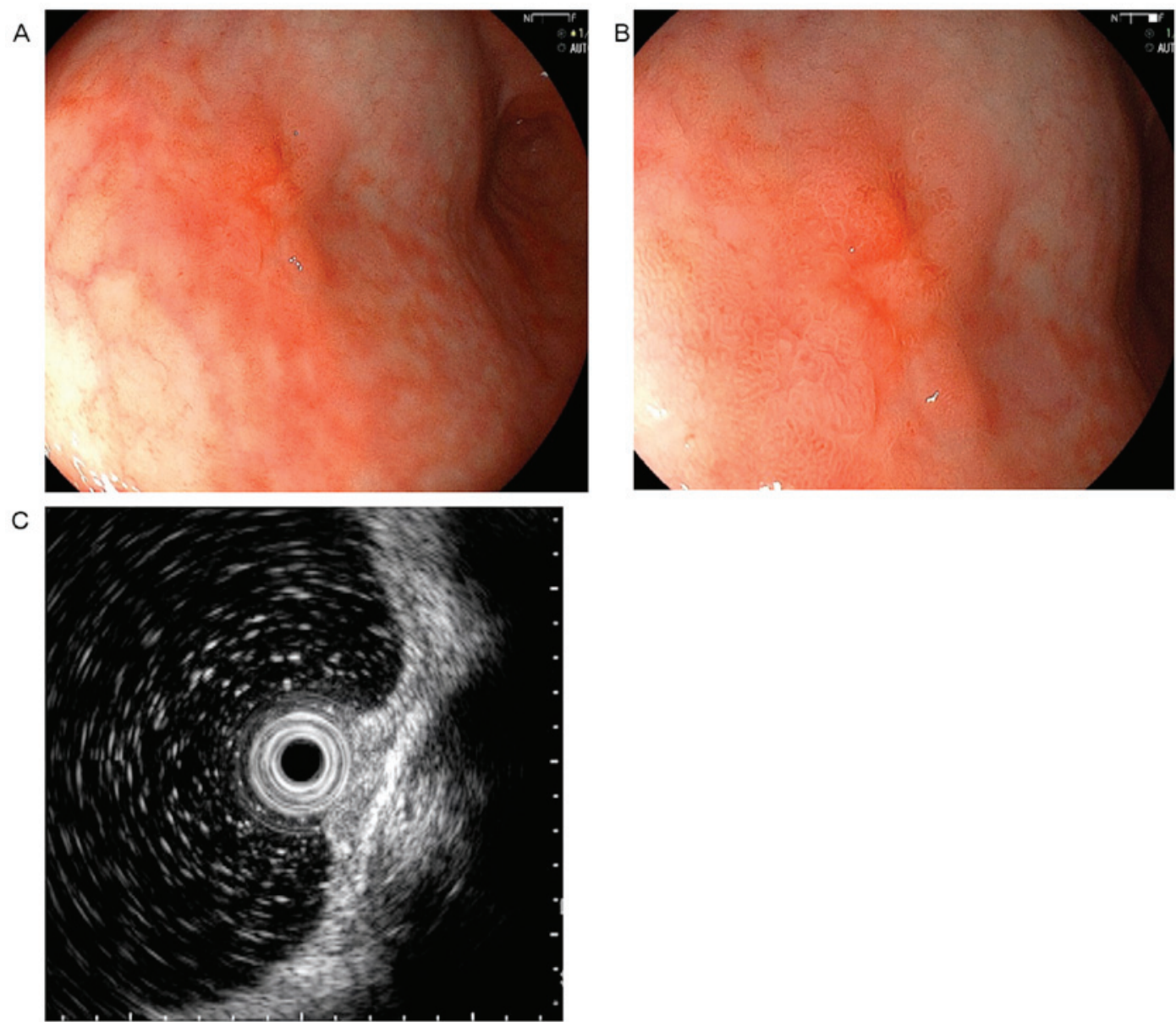

Figure 4. (A and B) Esophagogastroduodenoscopy revealed a superficial elevated lesion with a central depression $10 \mathrm{~mm}$ in size located in the lower body of the stomach. (C) Endoscopic ultrasonography confirmed that the tumor was confined to the mucosa.

A

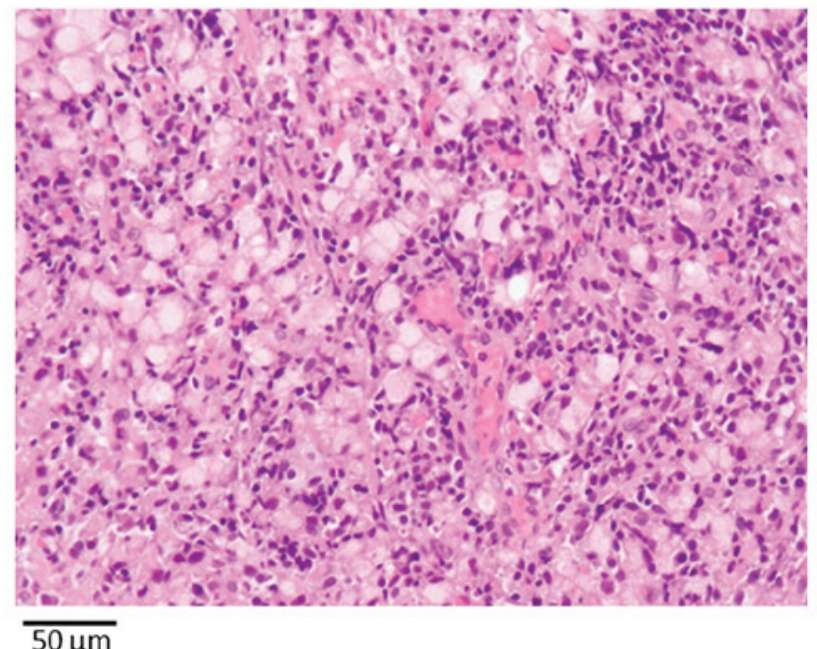

B

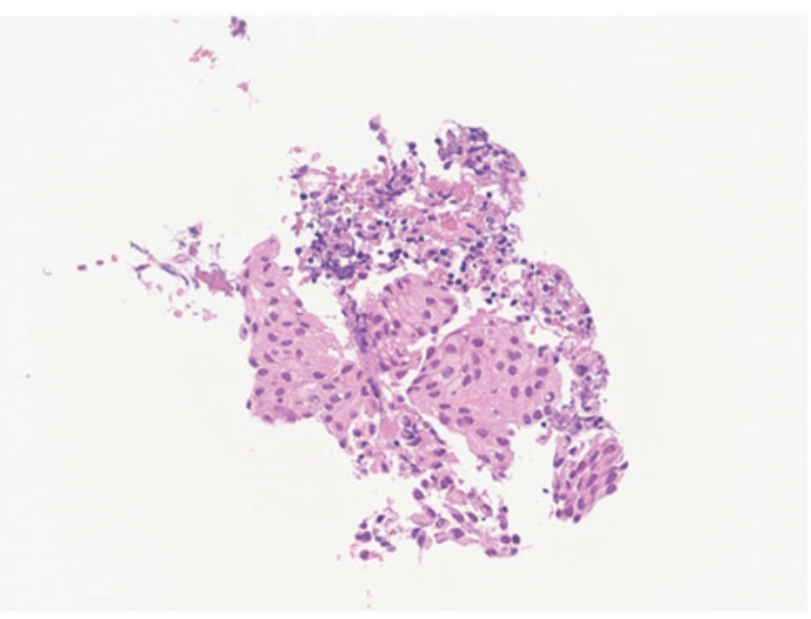

$100 \mu \mathrm{m}$

Figure 5. (A) Gastric biopsy: Histological examination demonstrated a signet-ring cell carcinoma on biopsy (hematoxylin and eosin stain). (B) Bone biopsy: Histological examination of the lumbar spine biopsy revealed cell aggregation, indicating signet-ring cell carcinoma (hematoxylin and eosin stain).

(SPIRITS) study, the median overall survival was 13.0 months with S-1/cisplatin (CDDP) therapy, and the standard first-choice treatment is S-1/CDDP therapy for advanced gastric cancer (22). The response rate of 3-weekly doses of nab-paclitaxel as second-line chemotherapy for advanced gastric cancer was reported to be $27.8 \%$, but recently, weekly nab-paclitaxel was non-inferior to weekly solvent-based paclitaxel in terms of overall survival $(23,24)$.

The usefulness of ramucirumab was recently reported. Wilke et al reported that the combination of ramucirumab 
with paclitaxel significantly increased overall survival compared with placebo plus paclitaxel (25). There have been some reports that $\mathrm{S}-1 / \mathrm{CDDP}$ or $\mathrm{S}-1$ plus oxaliplatin is effective for gastric cancer with bone metastasis, and these regiments might be promising for gastric cancer with bone metastasis (26-28). In our case, S-1/CDDP therapy was initiated without radiotherapy, owing to multiple bone metastasis. After the S-1/CDDP, the patient's LDH and serum calcium levels had decreased and pain had also alleviated, which effectively controlled his condition. But the effective period with S-1/CDDP was short; thus, the effectiveness of second-line therapy with nab-paclitaxel could not be confirmed. He could not receive third-line chemotherapy due to drug-induced interstitial pneumonia, and he died 120 days after consultation at our hospital. We believe that it is important to be able to continue chemotherapy to improve prognosis for patients with gastric cancer with bone metastasis, and further and larger clinical trials in the future will be important to achieving this goal.

We must be aware that there are cases of bone metastasis with signet-ring cell carcinoma from the time of the initial diagnosis, even at an early stage. Back pain are presented at the time of diagnosis or after treatment, PET-CT or whole-body bone scintigraphy should be performed.

\section{Acknowledgements}

Not applicable.

\section{Funding}

No funding was received.

\section{Availability of data and materials}

The datasets are available from the corresponding author on reasonable request.

\section{Authors' contributions}

IF and KM diagnosed, investigated and managed the patient. IF and TM contributed to the writing of the manuscript. TT and $\mathrm{JH}$ acquired the data, contributed clinical advice and critically reviewed the manuscript. TM, KM and SO acquired the data in the diagnostic imaging and pathological examination. All authors have read and approved the final version of the manuscript.

\section{Ethics approval and consent to participate}

Written informed consent was obtained from the patient.

\section{Patient consent for publication}

Written informed consent was obtained from the patient for the publication of the case details and associated images.

\section{Competing interests}

The authors declare that they have no competing interests.

\section{References}

1. Japanese Gastric Cancer Association: Japanese Classification of Gastric Carcinoma. 3rd English edition. Gastric Cancer 14: 101-112, 2011.

2. Shiraishi N, Inomata M, Osawa N, Yasuda K, Adachi Y and Kitano S: Early and late recurrence after gastrectomy for gastric carcinoma. Univariate and multivariate analyses. Cancer 89: 255-261, 2000.

3. Nakajima T: Gastric cancer treatment guidelines in Japan. Gastric Cancer 5: 1-5, 2002.

4. Santoro E, Garofalo A, Scutari FA, Carlini M, Zanarini T and Santoro E: Multicenter retrospective study of 3,024 patients operated on for stomach cancer in Italy. Epidemiology, surgical treatment and survival. Ann Gastroenterol Hepatol (Paris) 27: 167-171, 1991 (In French).

5. Park JM, Song KY, O JH, Kim WC, Choi MG and Park CH: Bone recurrence after curative resection of gastric cancer. Gastric Cancer 16: 362-369, 2013.

6. Shimada H, Okazumi S, Koyama M and Murakami K: Japanese gastric cancer association task force for research promotion: Clinical utility of ${ }^{18} \mathrm{~F}$-fluoro-2-deoxyglucose positron emission tomography in gastric cancer. A systematic review of the literature. Gastric Cancer 14: 13-21, 2011.

7. Japanese Gastric Cancer Association. Japanese gastric cancer treatment guidelines 2014 (Ver.4). Gastric Cancer 20: 1-19, 2017.

8. Yoshikawa K and Kitaoka H: Bone metastasis of gastric cancer. Jpn J Surg 13: 173-176, 1983.

9. Choi CW, Lee DS, Chung JK, Lee MC, Kim NK, Choi KW and Koh CS: Evaluation of bone metastasis by Tc-99m MDP imaging in patients with stomach cancer. Clin Nucl Med 20: 310-314, 1995.

10. Mori W, Adachi Y, Okabe H and Oota K: An analysis of 755 autopsied cases of malignant tumors. A statistical study of their metastasis. Gan No Rinsho 9: 351-374, 1963 (In Japanese).

11. Kobayashi M, Okabayashi T, Sano T and Araki K: Metastatic bone cancer as a recurrence of early gastric cancer-characteristics and possible mechanisms. World J Gastroenterol 11: 5587-5591, 2005.

12. Gurzu S, Jung I and Kadar Z: Aberrant metastatic behavior and particular features of early gastric cancer. APMIS 123: 999-1006, 2015.

13. Dassen AE, Lips DJ, Hoekstra CJ, Pruijt JFM and Bosscha K: FDG-PET has no definite role in preoperative imaging in gastric cancer. Eur J Surg Oncol 35: 449-455, 2009.

14. Kawamura T, Kusakabe T, Sugino T, Watanabe K, Fukuda T, Nashimoto A, Honma K and Suzuki T: Expression of glucose transporter-1 in human gastric carcinoma: Association with tumor aggressiveness, metastasis, and patient survival. Cancer 92: 634-641, 2001.

15. Yamada A, Oguchi K, Fukushima M, Imai Y and Kadoya M: Evaluation of 2-deoxy-2-[18F]fluoro-D-glucose positron emission tomography in gastric carcinoma: Relation to histological subtypes, depth of tumor invasion, and glucose transporter-1 expression. Ann Nucl Med 20: 597-604, 2006.

16. Alakus H, Batur M, Schmidt M, Drebber U, Baldus SE, Vallböhmer D, Prenzel KL, Metzger R, Bollschweiler E, Hölscher AH and Mönig S: Variable 18F-fluorodeoxyglucose uptake in gastric cancer is associated with different levels of GLUT-1 expression. Nucl Med Commun 31: 532-538, 2010.

17. Yoshioka T, Yamaguchi K, Kubota K, Saginoya T, Yamazaki T, Ido T, Yamaura G, Takahashi H, Fukuda H and Kanamaru R: Evaluation of 18F-FDG PET in patients with advanced, metastatic, or recurrent gastric cancer. J Nucl Med 44: 690-699, 2003.

18. Ma DW, Kim JH, Jeon TJ, Lee YC, Yun M, Youn YH, Park H and Lee SI: ${ }^{18} \mathrm{~F}$-fluorodeoxyglucose positron emission tomography-computed tomography for the evaluation of bone metastasis in patients with gastric cancer. Dig Liver Dis 45: 769-775, 2013

19. Kawanaka Y, Kitajima K, Fukushima K, Mouri M, Doi H, Oshima T, Niwa H, Kaibe N, Sasako M, Tomita T, et al: Added value of pretreatment (18)F-FDG PET/CT for staging of advanced gastric cancer: Comparison with contrast-enhanced MDCT. Eur J Radiol 85: 989-995, 2016.

20. Kang SH, Kim JI, Moon HS, Kang HM, Kim SH, Seong JK, Lee BS, Jeong HY, Song KS, Noh SM, et al: Overt bone marrow metastasis from early gastric cancer. Endoscopy 40: E34-E35, 2008. 
21. Hironaka SI, Boku N, Ohtsu A, Nagashima F, Sano Y, Muto M Fujii T, Tajiri $\mathrm{H}$ and Yoshida S: Sequential methotrexate and 5 -fluorouracil therapy for gastric cancer patients with bone metastasis. Gastric Cancer 3: 19-23, 2000.

22. Koizumi W, Narahara H, Hara T, Takagane A, Akiya T, Takagi M Miyashita K, Nishizaki T, Kobayashi O, Takiyama W, et al: S-1 plus cisplatin versus S-1 alone for first-line treatment of advanced gastric cancer (SPIRITS trial): A phase III trial. Lancet Oncol 9: 215-221, 2008.

23. Sasaki Y, Nishina T, Yasui H, Goto M, Muro K, Tsuji A, Koizumi W, Toh Y, Hara T and Miyata Y: Phase II trial of nanoparticle albumin-bound paclitaxel as second-line chemotherapy for unresectable or recurrent gastric cancer. Cancer Sci 105: 812-817, 2014.

24. Shitara K, Takashima A, Fujitani K, Koeda K, Hara H, Nakayama N, Hironaka S, Nishikawa K, Makari Y, Amagai K, et al: Nab-paclitaxel versus solvent-based paclitaxel in patients with previously treated advanced gastric cancer (ABSOLUTE): An open-label, randomised, non-inferiority, phase 3 trial. Lancet Gastroenterol Hepatol 2: 277-287, 2017.

25. Wilke H, Muro K, Van Cutsem E, Oh SC, Bodoky G, Shimada Y, Hironaka S, Sugimoto N, Lipatov O, Kim TY, et al: Ramucirumab plus paclitaxel versus placebo plus paclitaxel in patients with previously treated advanced gastric or gastro-oesophageal junction adenocarcinoma (RAINBOW): A double-blind, randomised phase 3 trial. Lancet Oncol 15: 1224-1235, 2014.
26. Woo Y, Fujisaki S, Takashina M, Tomita R, Sakurai K and Takayama T: A case of metastases to the bone, skin, and ovary from gastric cancer occurring more than eight years after distal gastrectomy. Gan To Kagaku Ryoho 44: 1571-1573, 2017 (In Japanese).

27. Takishita C, Yajima K, Iwasaki Y, Ohashi M, Iwanaga T and Oohinata R: A case of early gastric cancer with multiple synchronous bone metastases treated complete response with S-1+CDDP. Gan To Kagaku Ryoho 41: 2611-2614, 2014 (In Japanese)

28. Choi YJ, Kim DH, Han HS, Han JH, Son SM, Kim DS and Yun HY: Long-term survival after gastrectomy and metastasectomy for gastric cancer with synchronous bone metastasis. World J Gastroenterol 24: 150-156, 2018. Attribution-NonCommercial-NoDerivatives 4.0 International (CC BY-NC-ND 4.0) License. 